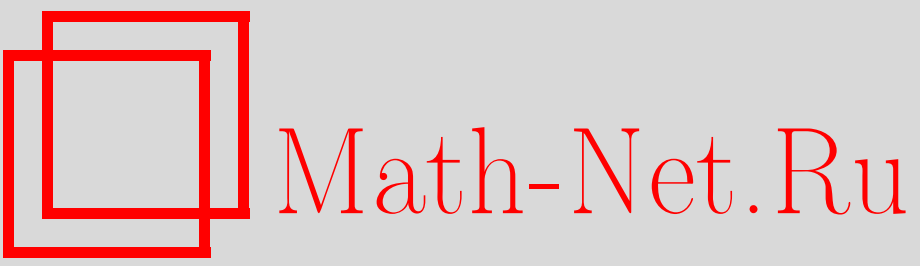

С. П. Суетин, О динамике "блуждающих" нулей полиномов, ортогональных на нескольких отрезках, УМН, 2002, том 57, выпуск 2, 199-200

DOI: https://doi.org/10.4213/rm507

Использование Общероссийского математического портала Math-Net.Ru подразумевает, что вы прочитали и согласны с пользовательским соглашением

http://www.mathnet.ru/rus/agreement

Параметры загрузки:

IP : 54.224 .60 .19

26 апреля 2023 г., 08:37:41 


\title{
О ДИНАМИКЕ “БЛУЖДАЮЩИХ" НУЛЕЙ ПОЛИНОМОВ, ОРТОГОНАЛЬНЫХ НА НЕСКОЛЬКИХ ОТРЕЗКАХ
}

\author{
С. П. Суетин
}

1. Рассмотрим функции вида $f=\widehat{\mu}+r$, где $\widehat{\mu}(z)=\int_{E} \frac{d \mu(x)}{z-x}, \mu$ - положительная мера с носителем $E \Subset \mathbb{R}, r \in \mathbb{C}(z)$ - комплексная рациональная функция, голоморфная на $E, r(\infty)=0$ $(f-$ "рациональное возмущение" $\widehat{\mu})$.

Функции $\widehat{\mu}$ были введены П. Л. Чебышевым [1] при изучении им формальных разложений лорановских рядов $\frac{c_{1}}{z}+\frac{c_{2}}{z^{2}}+\cdots$ в непрерьвные дроби вида $C(z):=\frac{b_{1}}{z-a_{1}}-\frac{b_{2}}{z-a_{2}}-\cdots$. Знаменатели $Q_{n}=Q_{n}(z ; \widehat{\mu})$ подходящих дробей $P_{n} / Q_{n}$ для $C(z)$ оказьваются многочленами, ортогональными по мере $\mu$. Для $\widehat{\mu}$ теорема Маркова [2] утверждает сходимость последовательности $\left\{P_{n} / Q_{n}\right\}$ к $\widehat{\mu}$ равномерно внутри области $\overline{\mathbb{C}} \backslash[a, b]$, где $[a, b]=\widehat{E}$ - выпуклая оболочка $E$. Тот факт, что в теореме речь идет о сходимости вне $\widehat{E}$, а не в дополнении к $E$, не случаен: если $E$ состоит из нескольких отрезков, то в лакунах между этими отрезками могут располагаться нули полинома $Q_{n}$. Дробь $P_{n} / Q_{n}$ есть в точности диагональная аппроксимация Паде $[n / n]_{\widehat{\mu}}$ функции $\widehat{\mu}$ в точке $z=\infty$; при нормировке $Q_{n}(z)=z^{n}+\cdots$ выполняется соотношение: $R_{n}(z):=\left(Q_{n} \widehat{\mu}-P_{n}\right)(z)=O\left(1 / z^{n+1}\right), z \rightarrow \infty$. Функция $R_{n}=R_{n}(z ; \widehat{\mu})$ назьвается функцией остатка. Если $E$ состоит из конечного числа отрезков, то в "типичной" ситуации предельные точки нулей последователшности $\left\{Q_{n}\right\}$ - это в точности весь отрезок $[a, b]$. С другой стороны, по некоторой подпоследовательности $\Lambda$ нули $Q_{n}$ не имеют предельных точек в лакунах между отрезками; тем самым, по этой подпоследовательности диагоналшные аппроксимации Паде $\left\{[n / n]_{\widehat{\mu}}\right\}_{n \in \Lambda}$ сходятся к $\widehat{\mu}$ равномерно внутри $\overline{\mathbb{C}} \backslash E$, а не толшко внутри $\overline{\mathbb{C}} \backslash \widehat{E}$ как в теореме Маркова (см. [3]).

Аналогичньй результат о существовании равномерно сходящейся подпоследовательности диагональных аппроксимаций Паде получен [3] и для $f=\widehat{\mu}+r$. В этом случае $Q_{n}(z ; f)$ ортогональны относительн меры $\mu+\sum_{j=1}^{m} \lambda_{j} \delta_{a_{j}}$, где $a_{j}$ - полюсы $r, a_{j}, \lambda_{j} \in \mathbb{C}$. Последовательность $\left\{[n / n]_{f}\right\}_{n \in \mathbb{N}}$ сходится к $f$ по (логарифмической) емкости, причем, препятствием к равномерной сходимости всей последовательности $\left\{[n / n]_{f}\right\}$ является "неопределенное" поведение части нулей $Q_{n}(z ; f)$ (полюсов $[n / n]_{f}$ ). В терминах гармонических мер отрезков носителя Рахманов [4] получил систему уравнений, описьвающих динамику таких нулей в зависимости от $n$. В случае одной лакуны единственное уравнение задает некоторую кривую $\gamma$, соединяющую оба отрезка и расположенную в $\overline{\mathbb{C}} \backslash E$. Единственный в этом случае нуль $\widetilde{z}_{n}$ полинома $Q_{n}(z ; f)$ с "неопределенным" поведением фактически "блуждает" около этой кривой: все предельные точки последователшности $\left\{\widetilde{z}_{n}\right\}$ лежат на $\gamma$. В “типичной” ситуации каждая точка $\gamma$ - предельная точка последовательности $\left\{\widetilde{z}_{n}\right\}$. Для произвольного числа лакун из уравнений Рахманова не удается извлечь явного описания динамики блуждающих нулей полиномов $Q_{n}(z ; f)$.

В настоящей заметке приводятся интегр ируемые дифференциальные уравнения, описывающие динамику таких нулей полиномов $Q_{n}(z ; f)$ в случае произвольног числа лакун.

2. Пусть $E$ состоит из конечного числа отрезков $E_{j}=\left[e_{2 j-1}, e_{2 j}\right], j=1, \ldots, \mathrm{g}+1$; $h(z)=z^{2 \mathrm{~g}+2}+\cdots-$ полином с нулями в точках $\left\{e_{k}\right\}$. Ветвь корня $\sqrt{h(z)}$ выберем так, что $\sqrt{h(z)} / z^{\mathrm{g}+1} \rightarrow 1$ при $z \rightarrow \infty$. Пусть мера $\mu$ имеет вид $d \mu(x)=\rho(x) d x / i \sqrt{h(x+i 0)}, x \in E$, где функция $\rho(x) \neq 0$ и удовлетворяет условию Гёльдера на $E$. Рассмотрим гиперэллиптическую риманову поверхность $\mathfrak{R}: w^{2}=h(z)$ рода $\mathrm{g}$, которая реализована как двулистное разветвленное накрытие римановой сферы $\overline{\mathbb{C}}$ таким образом, что переход с одного листа на другой осуществляется по верхнему $E_{j}^{+}$и нижнему $E_{j}^{-}$берегам отрезков $E_{j}$. Каждой точке $z \neq e_{k}$ на $\overline{\mathbb{C}}$ соответствуют две точки $z^{(1)}=(z, \sqrt{h(z)})$ и $z^{(2)}=(z,-\sqrt{h(z)})$ на $\Re ; D^{(1)}$ и $D^{(2)}-$ два листа поверхности, склеенные по $E^{+} \cup E^{-}$; функция $w=w(\mathbf{z}), \mathbf{z}=z^{(1)}$ или $z^{(2)}$, однозначна

Работа вьполнена при финансовой поддержке РФФИ (грант № 02-01-00564), Программы поддержки ведущих научных школ (грант № 00-15-96132) и фонда INTAS (грант № 00-272). 
на $\mathfrak{R}$. Определим [5] на $\mathfrak{R}$ базис гомологий, состоящий из $a$ и $b$-циклов, и соответствующий $a$-нормированный базис голоморфных абелевых дифференциалов $d W_{k}: \oint_{\mathbf{a}_{j}} d W_{k}(\boldsymbol{\zeta})=\delta_{k j}$, $k, j=1, \ldots, \mathrm{g}$. Матрица $\left\|B_{k j}\right\|_{k, j=1, \ldots, \mathbf{g}}, B_{k j}=\oint_{\mathbf{b}_{j}} d W_{k}(\boldsymbol{\zeta})$, является матрицей Римана. Функции $W_{k}(\mathbf{z})=\int_{e_{2 \mathrm{~g}+2}}^{\mathbf{z}} d W_{k}(\boldsymbol{\zeta})$ определены на $\mathfrak{R}$ однозначно по модулю их $a$ и $b$-периодов; функции $W_{k}^{\prime}(\mathbf{z})=p_{k}(z) / w(\mathbf{z})$ однозначны на $\mathfrak{R}, p_{k} \in i \mathbb{R}[z]$. Пусть $\omega_{j}(z)$ - гармоническая мера отрезка $E_{j}$ относительно области $D=\overline{\mathbb{C}} \backslash E$ в точке $z \in D, j=1, \ldots$, g.

3. Рассмотрим следующую систему дифференциалшных уравнений относителшно набора точек $\mathbf{z}_{1}(t), \ldots, \mathbf{z}_{\mathbf{g}}(t), t \in \mathbb{R}_{+}$, на $\mathfrak{R}$ :

$$
\dot{z}_{j}=-2 \frac{w\left(\mathbf{z}_{j}\right)}{\prod_{l \neq j}\left(z_{j}-z_{l}\right)} \int_{e_{2 \mathrm{~g}+2}}^{\infty} \frac{\prod_{l \neq j}\left(x-z_{l}\right)}{w(x)} d x, \quad j=1, \ldots, \mathrm{g}
$$

(точка означает производную по $t$ ). Пусть $\boldsymbol{\Gamma}_{j}$ - траектория, которую описывает на $\mathfrak{R} j$-я точка $\mathbf{z}_{j}(t)$. Проекция $\Gamma_{j}$ кривой $\boldsymbol{\Gamma}_{j}$ на $\mathbb{C}-$ замкнутая кривая, симметричная относителшно $\mathbb{R}$. Расположение $\boldsymbol{\Gamma}_{j}$ зависит от "начальных" условий $\mathbf{z}_{j}(0)$; в частности, если все $z_{j}(0) \in\left[e_{2 j}, e_{2 j+1}\right]$, то $\Gamma_{j}=\left[e_{2 j}, e_{2 j+1}\right]$.

Уравнения $(*)$ полностью определяют динамику блуждающих нулей полиномов $Q_{n}(z ; f)$ в следующем смысле. Пусть $\mathbf{z}_{1}(t), \ldots, \mathbf{z}_{\mathbf{g}}(t)$ - решения $(*)$ с некоторыми началњными условиями, $\boldsymbol{\Gamma}_{j}$ - соответствующая $\mathbf{z}_{j}(t)$ траектория. При фиксированном $j \in\{1, \ldots, \mathrm{g}\}$ рассмотрим последовательность $\left\{\mathbf{z}_{j}(n)\right\} \subset \boldsymbol{\Gamma}_{j}, n \in \mathbb{N}$.

Теорема 1. Если по некоторой подпоследовательности $\Lambda_{1} \subset \mathbb{N}$ все $\mathbf{z}_{j}(n) \in D^{(1)}$, то для $n \in \Lambda_{1}$ функиия остатка $R_{n}$ имеет блуждающий нуль $\widehat{z}_{n}$, которьй бесконечно близок $к z_{j}(n)$ при $n \rightarrow \infty$. Если по некоторой подпоследовательности $\Lambda_{2} \subset \mathbb{N}$ все $\mathbf{z}_{j}(n) \in D^{(2)}$, то для $n \in \Lambda_{2}$ полином $Q_{n}$ имеет блуждающий нуль $\widetilde{z}_{n}$, который бесконечно близок $\kappa z_{j}(n)$ при $n \rightarrow \infty$.

Таким образом, задача об асимптотическом поведении блуждающих нулей сводится к изучению свойств решений $\mathbf{z}_{1}(t), \ldots, \mathbf{z}_{\mathbf{g}}(t)$ системы $(*)$.

Tеорема 2. Система $(*)$ :

1) интегрируется в явном виде с помощью преобразования Абеля; решения системы - условно периодические функции переменной $t$ с периодами $\omega_{1}(\infty), \ldots, \omega_{\mathrm{g}}(\infty)$;

2) әквивалентна системе уравнений Рахманова;

3) в пределе при е $2 \mathrm{~g}+2 \rightarrow \infty$ переходит в систему уравнений Дубровина (см. [6])

$$
\dot{z}_{k}=\frac{4 \sqrt{-h\left(z_{k}\right)}}{\prod_{j \neq k}\left(z_{k}-z_{j}\right)}, \quad k=1, \ldots, \mathrm{g},
$$

описывающую динамику нулей конечно-зонных решений уравнения Шрёдингера.

В “типичном" случае множество $\left(\mathbf{z}_{1}(n), \ldots, \mathbf{z}_{\mathrm{g}}(n)\right)$ всюду плотно на $\boldsymbol{\Gamma}_{1} \times \cdots \times \boldsymbol{\Gamma}_{\mathbf{g}}$.

\section{СПИСОК ЛИТЕРАТУРЫ}

[1] П. Л. Чебышев // Полное собрание сочинений. Т. ІІ.. М.-Л.: Изд-во АН СССР, 1948. C. 103-126. [2] А.А. Марков // Избранные труды по теории непрерывных дробей и теории функций, наименее уклоняющихся от нуля. М.: Гостехиздат, 1948. С. 106-119. [3] С. П. Суетин // Матем. сб. 2000. Т. 191. № 9. С. 81-114. [4] Е. А. Рахманов // Матем. сб. 1977. Т. 104 (146). № 2. С. 271-291. [5] Э. И. Зверович // УМН. 1971. Т. 26. №1. С. 113-180. [6] Б. А. Дубровин // Функц. анализ и его прил. 1975. Т. 9. № 3. С. 41-52. 